\title{
Validation of a self-administered olfactory and gustatory test for the remotely evaluation of COVID-19 patients in home quarantine
}

\author{
Luigi Angelo Vaira, $\mathrm{MD}^{1}$ \\ ${ }^{1}$ University Hospital of Sassari
}

April 28, 2020

\begin{abstract}
Background. Chemosensitive disorders are very frequent in the early stages of COVID-19 and in paucisymptomatic cases. These patients are typically placed in home quarantine. This study has the aim of validating a new olfactory and gustatory objective evaluation test in these patients.

Methods. Thirty-three home-quarantined COVID-19 patients have undergone a self-administered chemosensitive test the day before the control swab. On this occasion, the patients underwent operator-administered already validated tests. The results were finally compared.

Results. The differences between the results of the two tests were not significant for both the olfaction $(\mathrm{P}=0.201)$ and the taste $(\mathrm{P}=0.180)$

Conclusion. The olfactory and gustatory evaluation by self-administered test can be considered a valid tool, fundamental for obtaining objective qualitative and quantitative data on the extent of chemosensitive disorders in home-quarantined COVID-19 patients.
\end{abstract}

Luigi Angelo Vaira ${ }^{*}$, MD; Giovanni Salzano ${ }^{2}$, MD; Marzia Petrocelli ${ }^{3}$, MD; Giovanna Deiana ${ }^{4}$, MD; Francesco Antonio Salzano ${ }^{5}$, MD; Giacomo De Riu ${ }^{1}$ MD, FEBOMFS.

1 Maxillofacial Surgery Operative Unit, University Hospital of Sassari, Viale San Pietro 43/B, 07100 Sassari, Italy.

2 Maxillofacial Surgery Operative Unit, University Hospital of Naples "Federico II", Via Pansini 5, 80131 Naples, Italy.

3 Maxillofacial Surgery Operative Unit; Bellaria and Maggiore Hospital, AUSL Bologna, Italy.

4 Department of Medical, Surgical and Experimental Sciences, University of Sassari, Viale San Pietro, 07100 Sassari, Italy.

5 Department of Medicine, Surgery and Dentistry, "Scuola Medica Salernitana", University of Salerno, 84081 Baronissi (Salerno), Italy

*Corresponding Author:

Luigi Angelo Vaira

Viale San Pietro 43/B

e-mail: luigi.vaira@gmail.com

tel.: +393401846168

fax: +39079229002 
Keywords: COVID-19; anosmia; ageusia; gustatory function; olfactory function.

\begin{abstract}
Background. Chemosensitive disorders are very frequent in the early stages of COVID-19 and in paucisymptomatic cases. These patients are typically placed in home quarantine. This study has the aim of validating a new olfactory and gustatory objective evaluation test in these patients.
\end{abstract}

Methods. Thirty-three home-quarantined COVID-19 patients have undergone a self-administered chemosensitive test the day before the control swab. On this occasion, the patients underwent operator-administered already validated tests. The results were finally compared.

Results. The differences between the results of the two tests were not significant for both the olfaction $(\mathrm{P}=$ $0.201)$ and the taste $(\mathrm{P}=0.180)$.

Conclusion. The olfactory and gustatory evaluation by self-administered test can be considered a valid tool, fundamental for obtaining objective qualitative and quantitative data on the extent of chemosensitive disorders in home-quarantined COVID-19 patients.

\title{
INTRODUCTION
}

Olfactory and gustatory dysfunctions are proving to be frequent symptoms of severe acute respiratory syndrome coronavirus 2 (SARS-CoV-2) infection. The first European reports on coronavirus disease 19 (COVID19) patients are detecting a frequency of these symptoms ranging between $19.4 \%$ and $88 \%$ [1-5]. Particularly, chemosensitive dysfunctions appear to be common in the early stages of infection from severe acute respiratory syndrome coronavirus 2 (SARS-CoV-2) and in paucisymptomatic patients [1,2]. However, all the reports published so far are anamnestic or observational and the literature lacks objective studies that evaluate the gustatory and olfactory function in these patients. Assessing the patient in the hospital is difficult due to the entry restrictions into the COVID-19 Departments and to the fact that the most serious cases that require hospitalization, are generally uncooperative. Moreover, hospitalized patients are generally in the late stages of the infection and it would instead be interesting to acquire objective information on asymptomatic or initial cases, in order to find trigger symptoms that can allow early identification and isolation of suspect cases. Generally, the latter patients are in home quarantine. Therefore, checking and evaluating them at home presents almost insurmountable logistical problems during an emergency health situation like the one we are facing. An objective and validated self-administered test would be a valuable tool for the remote evaluation of these patients.

Psycho-physiological tests represent a workhorse in the objective evaluation of the olfactory and gustatory functions as they are easy to perform, standardized, validated and repeatable $[6,7]$. These tests are generally based on the blindness of the assessed patient, which guarantees the reliability and validity of the results. Clearly, this basic principle cannot be insured if the subject, in home isolation, is asked to self-administer the test.

The aim of this work is to overcome this bias, developing a new self-administered psycho-physiological olfactory and gustatory test that could be used to evaluate COVID-19 patients in home quarantine.

The same group of patients, after the completion of the proposed self-administered test, underwent direct clinical evaluation with widely used and validated tests for anosmia and ageusia. Then, the results of both kind of tests were statistically compared to validate the self-administered evaluation protocol.

\section{MATERIALS AND METHODS}

The study was conducted between April $9^{\text {th }}$ and $10^{\text {th }}$ 2020, on home-quarantined SARS-CoV-2 positive patients, followed by the Surveillance and Prevention Department of the University of Sassari, University of Salerno and Bellaria-Maggiore Hospital of Bologna. All patients belonged to the healthcare staff of the involved hospitals (i.e. doctors, nurses, auxiliary staff). 
The inclusion criteria were: home quarantined adults, age $>18$ years old, rhino-pharyngeal swab positive for SARS-CoV-2 infection, scheduled control swab, voluntary participation in the study.

Previous surgery or radiotherapy in the oral and nasal cavities, pre-existing manifestation of smell and taste alterations, history of head trauma, allergic rhinitis, chronic rhinosinusitis and psychiatric or neurological disorders were considered as exclusion criteria.

The study protocol was approved by an independent ethics committee ( $\mathrm{n}^{\circ} 378-2020$-OSS-AUSLBO).

The day before the scheduled control swab, patients in home quarantine were asked to participate in the study. In case of acceptance, some general information was collected for all subjects: gender, age, job position, comorbidity or conditions that could be cause of exclusion, which symptoms presented and when these symptoms started, when positivity to the first nasopharyngeal swab was confirmed. All patients were carefully investigated for the previous or current presence of chemosensitive disorders during the SARS-CoV-2 infection. Finally, the test methodology was explained and patients were asked to collect 7 common household odorants and prepare the 4 basic flavored necessary solutions, thus contacting the operator again to perform the telephone test.

The next morning at the hospital, before the control swab, patient's chemosensitive functions were directly evaluated by a different trained operator.

\section{Patient-administrated olfactory and gustatory test}

Both olfaction and taste were assessed in all the patients. The olfactory threshold was determined using solutions with a decreasing concentration of denatured ethyl-alcohol. Ethyl-alcohol has an olfactory threshold 10 times lower than N-butyl-alcohol [8], therefore the patient was asked to prepare a $40 \%$ in $100 \mathrm{~mL}$ water solution (bottle 0) using a using a syringe or a graduated container. In the following bottles (from 1 to 8), the patients diluted a part of the previous solution with two parts of water, thus obtaining solutions with consequent 1:3 dilutions. Finally, a control bottle was filled with water only. The test was performed by asking the patient to smell the alcohol-containing bottle, starting with the less concentrated (bottle 8), reporting if he found any differences compared to the control bottle. In case of a negative answer, the examination continued with the consequent bottle.

The discriminative test was performed asking the patient to find some commonly used odorants [Table 1]. Therefore, the subject smelled an odorant for each of the 7 groups providing an assessment of the discriminative ability from 0 (no discrimination) to 10 (normal discrimination). The discriminatory score was obtained from the average of the ratings of the 7 odorants.

The threshold and identification test scores, after being analyzed separately, were finally converted into a composite score. The scoring system is shown in table 2 [Table 2].

For the evaluation of the gustatory function, the patient was asked to prepare four solutions, one for each primary taste [9-11]:

- Salted solution: $30 \mathrm{~g}$ of table salt in 1 liter of water.

- Sweet solution: $60 \mathrm{~g}$ of refined sugar in 1 liter of water.

- Sour solution: $90 \mathrm{~mL}$ of $100 \%$ lemon juice in 1 liter of water.

- Bitter solution: unsweetened decaffeinated coffee.

The patient was asked to put in mouth a tea spoon of each solution, reporting the quality of taste perception, scoring it from 0 (ageusia) to 10 (normal perception). The bitter solution comes always the last [9-10]. The overall test score was obtained by the average of the values reported for each of the primary tastes. The scoring system is reported in table 3 [Table 3].

Operator-administered olfactory and gustatory test

The Connecticut Chemosensorial Clinical Research Center (CCCRC) orthonasal olfaction test [12-16] was used to evaluate the olfactory function. As required by the test protocol, olfactory threshold was determined 
by means of $60 \mathrm{~mL}$ deionized waters solutions, with increasing concentration of N-butyl-alcohol. The most concentrated bottle (bottle 0) presented $4 \%$ dilution. The other 8 bottles (bottles 1-8) contained decreasing concentrations with consequent 1:3 dilutions. For each threshold step, two identical squeezable bottles were presented to the patient: one containing the N-butanol solution, from the major to the minor dilution, and the other filled with deionized water. The test was conducted as previously described for both nostrils, and the average value determined the overall score [17].

Ten well-known Italian odorants were used to assess the olfactory discriminative function: chocolate (Nutella, Ferrero, Italy), coffee, Baby powder (Manetti \& Roberts, Florence, Italy), Vicks-VapoRub (Proctor \& Gamble, Cincinnati, OH, USA), ammonia, fruit-flavored chewing gum (Perfetti Van Melle Italia S.r.l, Lainate, Italy), ketchup (Heinz, Pittsburgh, PA, USA), orange, soap (Ivory, Proctor \& Gamble, Cincinnati, OH, USA) and black pepper. The odorants were presented one at a time, in identical and opaque $180 \mathrm{~mL}$ containers covered by gauze. Therefore, the patient had to identify the odorant on a 20 items list containing the 10 test samples and 10 distractors.

The threshold and discriminative test scores were finally converted into the CCCRC composite score. The scoring system is shown in Table 2 [Table 2].

As regards the gustatory function assessment, the primary taste solutions were identical to those used in the telephone test and already proposed by other authors [9,10]. As the only variation, at the hospital, deionized water was used as solvent and control instead of normal drinking water. One at a time, approximately $1 \mathrm{ml}$ of each basic flavor was put on the patient's tongue with a cotton swab, taking care to test the bitter last. Therefore, the patient had to indicate the perceived taste: salty, acid, sweet, bitter or neutral. Depending on the number of primary tastes perceived, the score could vary between 0 and 4 . The scoring system is reported in table 3 [Table 3$]$.

\section{Statistical analysis}

Statistical analysis was performed using SPSS 26.0 (IBM, Armonk, NY, USA). Categorical variables are reported in numerals and percentages of the total. Descriptive statistics for quantitative variables are given as the mean +- standard deviation (SD). Statistical analysis of score differences reported by the patients in the two evaluations was performed by Wilcoxon signed-rank test for paired data. The level of statistical significance was set at P [?] 0.05 with a $95 \%$ confidence interval.

\section{RESULTS}

The study population included 33 COVID-19 patients (11 males, 22 females, mean age 51.8 years old) followed by the Security and Prevention Departments of the University Hospital of Sassari, University Hospital of Salerno and Bologna Maggiore-Bellaria Hospital. Table 4 provides a framework summary of patient general and clinical features [Table 4].

During the course of the infection, current or regressed chemosensitive dysfunctions were reported by 21 patients $(63.6 \%)$. In detail, 13 patients (39.4\%) reported combined gustatory and olfactory disorders. Four patients $(12.1 \%)$ presented isolated reduction in taste, while isolated hypo/anosmia was reported in other 4 patients $(12.1 \%)$. At the time of the telephone evaluation, 11 out of 21 patients $(52.4 \%)$ reported complete regression of chemosensitive symptoms.

Anamnestically, 17 patients (51.5\%) reported having or having had olfactory disorders during infection, including 13 cases of anosmia and 4 cases of hyposmia. Both the self-administered test and that performed by the operator showed normosmia in 8 patients, various degree hyposmia in 21 patients and complete anosmia in 4 cases. Compared to the CCCRC test, the telephone evaluation revealed more cases of moderate hyposmia (13 vs 9 ) and fewer of mild hyposmia (5 vs 9 ) [Chart 1].

Of the 17 patients (51.5\%) who complained gustatory dysfunction, 10 reported ageusia, 4 hypogeusia and 2 dysgeusia. The results of the telephone and outpatient evaluations are shown in chart 2 [Chart 2]. 
The olfactory $(\mathrm{P}=0.201)$ and taste $(\mathrm{P}=0.180)$ scores did not report any significant statistical difference between the self- and operator-administered evaluations [Table 5].

\section{DISCUSSION}

Early identification and isolation of infected patients is the most important therapeutic strategy for slowing the spread of SARS-CoV-2. It is therefore necessary to establish which are the most frequent symptoms in the initial and paucisymptomatic forms of COVID-19. In the first days of April we reported the high rates of olfactory and gustatory disturbances in these patients [1]. In the last two weeks, several other authors have reported the same clinical finding [2-5].

Recently, we were the first to objectively analyze the chemoreceptive functions in hospitalized COVID19 patients, confirming that these disturbances appear commonly in the first days of the infection and in paucisymptomatic cases [17]. A functional evaluation in these early stages of the disease would be very useful, to objectively establish the real incidence and extent of chemosensitive symptoms, but it is difficult to carry it out for at least two orders of reasons. First, these patients are generally not hospitalized and perform the evaluation at home is logistically impossible during the health crisis that we are facing. Secondly, in literature there are no psychophysiological or instrumental tests that can be remotely administered.

The tests we used in this study were created on the basis of already validated and widely used olfactory and gustatory evaluation protocols [9-16]. The scoring system was established so that the results of the two tests would be comparable.

The rates of taste and smell dysfunction during the infection (63.6\%) in the present case series were consistent with those of our previous analysis on hospitalized patients [17]. The differences between the results of the two tests were statistically insignificant, both for taste and olfaction. In general, non-blind patients remotely evaluated by telephone tend to underestimate the olfactory threshold. They tend to report perception for more diluted solutions. This bias is partially compensated by a tendency to obtain lower discriminative scores, due to the different evaluation mechanisms of the two tests. In fact, the patient-administered tests provide a quantitative assessment (from 1 to 10) of perception while the operator-administered tests provide a mere qualitative answer to forced identification choice. In this way, for example, even a patient with a mild or moderate hyposmia or hypogeusia could still be able to identify all the odors or tastes obtaining the maximum score on the outpatient tests. The score would instead be sub-normal if we asked the patient to quantitatively evaluate the extent of perception and not only his presence or absence. Overall, the results of the statistical analysis attest to the validity of auto-administered chemosensitive evaluation protocol.

Some possible limitations must however be mentioned. First, the study cohort was composed only of infected health personnel. Therefore, it was not a representative sample of the general population. The evaluated subjects were highly compliant for such a complex test, it is possible that the reliability of the test will decrease by extending enrollment to the general population, especially if elderly patients. For this reasons, the inclusion criteria should be carefully evaluated, limiting the assessment to very compliant subjects. Secondly, the self-administered test has proven to be very accurate in identifying patients with complete anosmia or ageusia, severe hyposmia and hypogeusia and in cases of normal chemopercective functions. On the other hand, it tends to underestimate some cases of moderate hyposmia and hypogeusia, downgrading them to mild forms [Chart 1, Chart 2]. Due to the logistical problems already reported, in this validation study, patients were evaluated in advanced stages of the clinical course (20.1 days from clinical onset, on average). For this reason, in many cases the chemosensitive disorder was already resolved and the severity of the residual dysfunction was very variable. On the basis of what has been reported by patients, it is reasonable to think that objective evaluation at an earlier stage would detect more serious or complete dysfunctions or, on the contrary, no functional alterations, rather than intermediate disturbances. In the early stages the test should be even more accurate.

\section{CONCLUSIONS}

Based on the results of this study on quarantined patients, the olfactory and gustatory evaluation by self- 
administered test can be considered a valid tool, fundamental for remotely obtaining qualitative and quantitative data on the extent of chemosensitive disorders. These data could lead to early detection and isolation of paucisymptomatic COVID-19 cases.

\section{FUNDING}

None declared

\section{CONFLICT OF INTEREST}

None of the authors has a financial interest in any of the products, devices or drugs mentioned in this manuscript.

ACNOWLEDGEMENT None declared

\section{REFERENCES}

[1] Vaira LA, Salzano G, Deiana G, De Riu G. Anosmia and ageusia: common findings in COVID-19 patients. Laryngoscope. 2020. Doi: 10.1002/lary.28692.

[2] Gane SB, Kelly C, Hopkins C. Isolated sudden onset anosmia in COVID-19 infection. A novel syndrome? Rhinology. 2020. Doi: 10.4193/Rhin20.114.

[3] Lechien JR, Chiesta-Estomba CM, De Siati DR et al. Olfactory and gustatory dysfunctions as a clinical presentation of mild-to-moderate forms of the coronavirus disease (COVID-19): a multicenter European study. Eur Arch Otorynolaryngol. 2020. Doi: 10.1007/s00405-020-05965-1

[4] Giacomelli A, Pezzati L, Conti F et al. Self-reported olfactory and taste disorders in SARS-CoV-2 patients: a cross-sectional study. Clin Infect Dis. 2020 Doi: 10.1093/cid/ciaa330.

[5] Hopkins C, Surda P, Kumar BN. Presentation of new onset anosmia during COVID-19 pandemic. Rhinology. 2020; Doi:https://doi.org/10.4193/Rhin20.116.

[6] Dory RL. Psychopysical testing of smell and taste function. Handb Clin Neurol. 2019;164:229-46.

[7] Spotten LE, Corish CA, Lorton CM et al. Subjective and objective taste and smell changes in cancer. Ann Oncol. 2017;28:969-84.

[8] Nagata Y. Measurement of odor threshold by triangle odor bag method. Odor Measurement Review. 2003;118:118-27.

[9] Massarelli O, Vaira LA, Biglio A, Gobbi R, Dell'aversana Orabona G, De Riu G. Sensory recovery of myomucosal flap oral cavity reconstructions. Head Neck. 2018;40:467-74.

[10] Pingel J, Ostwald J, Pau HW, Hummel T, Just T. Normative data for a solution-based taste test. Eur Arch Otorhinolaryngol. 2010;267:1911-7.

[11] Hwang CS, Kim JW, Al Sharhan SS, Kim JW, YOON JH, Kim CH. Development of a gustatory function test for clinical application in Koerean subjects. Yonsei Med J. 2018;59:325-30.

[12] Cain WS, Gent, J, Catalanotto FA, Goodspeed RB. Clinical evaluation of olfaction. Am J Otolaryngol. $1983 ; 4: 252-6$.

[13] Cain WS, Gent JF, Goodspeed RB, Leonard G. Evaluation of olfactory dysfunction in the Connecticut Chemosensory Clinical Research Center. Laryngoscope. 1988;98:83-8.

[14] Leon EA, Catalanotto FA, Werning JW. Retronasal and orthonasal olfactory ability after laryngectomy. Arch Otolaryngol Head Neck Surg. 2007;133:32-6.

[15] Aksoy C, Elsurer C, Artac H, Bozkurt MK. Evaluation of olfactory function in children with seasonal allergic rhinitis and its correlation with acoustic rhinometry. Int J Ped Otorhinolaryngol. 2018;113:188-91. 
[16] Veyseller B, Ozucer B, Karaaltin AB, Yildirim Y, Degirmenci N, Aksoy F, Ozturan O. Connecticut (CCCRC) olfactory test: normative values in 426 healthy volunteers. Indian J Otolaryngol Head Neck Surg. 2014;66:31-4.

[17] Vaira LA, Deiana G, Fois AG et al. Objective evaluation of anosmia and ageusia in COVID-19 patients: a single-center experience on 72 cases. Head Neck. 2020. Doi: 10.1002/HED.26204

\section{TABLE LEGEND}

\section{Hosted file}

Table_2.docx available at https://authorea.com/users/312328/articles/445021-validation-of-aself-administered-olfactory-and-gustatory-test-for-the-remotely-evaluation-of-covid-19-patientsin-home-quarantine

\section{Hosted file}

Table_3.docx available at https://authorea.com/users/312328/articles/445021-validation-of-aself-administered-olfactory-and-gustatory-test-for-the-remotely-evaluation-of-covid-19-patientsin-home-quarantine

\section{Hosted file}

Table_4.docx available at https://authorea.com/users/312328/articles/445021-validation-of-aself-administered-olfactory-and-gustatory-test-for-the-remotely-evaluation-of-covid-19-patientsin-home-quarantine

\section{Hosted file}

Table_5.docx available at https://authorea.com/users/312328/articles/445021-validation-of-aself-administered-olfactory-and-gustatory-test-for-the-remotely-evaluation-of-covid-19-patientsin-home-quarantine

\section{Hosted file}

Chart_1.tif available at https://authorea.com/users/312328/articles/445021-validation-of-a-selfadministered-olfactory-and-gustatory-test-for-the-remotely-evaluation-of-covid-19-patientsin-home-quarantine

\section{Hosted file}

Chart_2.tif available at https://authorea.com/users/312328/articles/445021-validation-of-a-selfadministered-olfactory-and-gustatory-test-for-the-remotely-evaluation-of-covid-19-patientsin-home-quarantine 